\title{
Fluorescence Correlation Spectroscopy of the Binding of Nucleotide Excision Repair Protein XPC-hHr23B with DNA Substrates
}

\author{
Y. Roche $\cdot$ D. Zhang $\cdot$ G. M. J. Segers-Nolten • \\ W. Vermeulen • C. Wyman • K. Sugasawa • \\ J. Hoeijmakers • C. Otto
}

Received: 28 March 2008 /Accepted: 26 May 2008 /Published online: 24 June 2008

(C) The Author(s) 2008

\begin{abstract}
The interaction of the nucleotide excision repair (NER) protein dimeric complex XPC-hHR23B, which is implicated in the DNA damage recognition step, with three Cy3.5 labeled 90-bp double-stranded DNA substrates (unmodified, with a central unpaired region, and cholesterol modified) and a 90-mer single-strand DNA was investigated in solution by fluorescence correlation spectroscopy. Autocorrelation functions obtained in the presence of an excess of protein show larger diffusion times $\left(\tau_{\mathrm{d}}\right)$ than for free DNA, indicating the presence of DNA-protein bound complexes. The fraction of DNA bound $(\theta)$, as a way to
\end{abstract}

Y. Roche $\cdot$ D. Zhang $\cdot$ G. M. J. Segers-Nolten $\cdot$ C. Otto $(\bowtie)$

Biophysical Engineering Group,

Faculty of Science and Technology,

$\mathrm{MESA}^{+}$Institute for Nanotechnology and BMTI,

University of Twente,

Enschede, The Netherlands

e-mail: c.otto@tnw.utwente.nl

W. Vermeulen $\cdot$ C. Wyman $\cdot$ J. Hoeijmakers

Medical Genetics Center,

Department of Cell Biology and Genetics,

Erasmus University,

Rotterdam, The Netherlands

K. Sugasawa

Laboratory of Cellular Physiology, Biosignal Research Center,

Kobe University,

Kobe, Japan

Present address:

Y. Roche

Institut National de la Recherche Agronomique,

UMR Plantes-Microbe-Environnement,

17 rue Sully, BP 86510 ,

21065 Dijon cedex, France describe the percentage of protein bound to DNA, was directly estimated from FCS data. A significantly stronger binding capability for the cholesterol modified substrate (78\% DNA bound) than for other double-stranded DNA substrates was observed, while the lowest affinity was found for the single-stranded DNA (27\%). This is in accordance with a damage recognition role of the XPC protein. The similar affinity of XPC for undamaged and 'bubble' DNA substrates (58\% and 55\%, respectively) indicates that XPC does not specifically bind to this type of DNA substrate comprising a large (30-nt) central unpaired region.

Keywords Fluorescence correlation spectroscopy .

Nucleotide excision repair - Oligonucleotides .

Macromolecules interaction

\section{Introduction}

In recent years, we have witnessed a rapid growth of interest in the field of single molecule detection and manipulation to investigate biological processes. Single molecule approaches are motivated by the possibility to follow processes that are otherwise hidden in the measured average properties of a system [1]. Hence, for complex biological reactions involving many components, active species may be rare but can still be identified and analyzed at the individual scale. Consequently, observation and analysis of protein-DNA interactions at the single molecule level can provide information on macromolecular interactions that cannot be obtained from standard bulk biochemical assays. In the case of single molecule spectroscopy, 
fluorescence detection offers good sensitivity and excitation and detection in a large spectral range. This is due to a wide variety of available fluorophores combined with progress in specific labeling techniques for proteins, nucleic acids and other biomolecules $[2,3]$. Confocal scanning fluorescence microscopy is sufficiently sensitive to allow observation of individual fluorescent molecules in solution as well as at surfaces, independent of interaction with a scanning probe, such as in near-field techniques like NSOM or AFM. Two main strategies can be followed to ensure the spatial, temporal or spectral isolation of individual species in order to study their behavior: (1) Fluorescence imaging of immobilized molecules in which the same molecule can be studied over time; (2) the emission bursts of a low number of molecules diffusing in solution, in which many individual molecules contribute to the signal [4].

Fluorescence correlation spectroscopy (FCS) is a method which relies on this second strategy $[5,6]$. It is based on the analysis of stochastic deviations in fluorescence intensity caused by any dynamic process that occurs while (individual) molecules are present in an open excitation volume created by a strongly focused laser beam. Detected fluorescence fluctuations are autocorrelated at different values of time-shift, or lag time $(\tau)$. The resulting autocorrelation function is directly proportional to the probability of detecting, on average, a photon at time $\tau$ if there were a detection event at time zero. Autocorrelation analysis gives insight in the processes that underlie these fluorescence fluctuations, such as triplet-states dynamics and diffusion of molecules through the detection volume, characterized by the diffusion time $\left(\tau_{\mathrm{d}}\right)$. Pioneering works on FCS were described in the early 1970s [7] but it was only in the 1990s that technological advances in detectors, laser stability, electronics and confocal microscopy, enabled single molecule detection by FCS [8]. Since then, the potential of FCS has been demonstrated from many investigations of biological systems at the single molecule level [9-11]. Among all possible applications, FCS is a powerful tool for assaying molecular interactions of biological importance, in dilute systems, with very high specificity. The method is well suited to study the binding of a small compound to larger molecular species. Indeed, if the small compound is labeled with a fluorescent dye, FCS can monitor its binding to a larger object from a decrease in diffusion rate. By analyzing the autocorrelation function it is possible to resolve the contributions from the fast diffusion of the free small labeled species and the slow diffusion of the bound complex and to determine the concentrations of both the unbound and bound fractions in the mixture [12]. FCS was extensively used to characterize DNA-protein binding complexes at the single molecule level, for example to reveal the oligomerization of $\mathrm{NtrC}$ protein on DNA [13], to characterize interaction of single stranded DNA with hexameric RepA DNA-helicase [14] and with RPA [15]. Wolcke et al. [16] showed the consistency between FCS and electrophoretic mobility shift assay (EMSA) measurements to demonstrate the sequencespecific binding of activated p53 to double-stranded DNA. Interestingly, mass sensitivity improvements to determine DNA-protein binding were proposed by combining FCS with high-speed capillary EMSA [17]. Recently, FCS was used to detect the binding of transcription factors in nuclear extracts of HeLa cells [18].

We investigate protein-DNA complexes involved in nucleotide excision repair (NER). NER is one of the most important mammalian DNA repair processes responsible for removal of damages that significantly distort the doublehelix conformation [19]. From biochemical methods much knowledge has been gained on the NER mechanism, which involves several proteins and includes recognition of the damage, its demarcation, dual incision around the lesion, release of a 24-32 nucleotide damage-containing DNA fragment, gap-filling, DNA synthesis and ligation. Although most of the proteins participating in a NER event are known, questions about the composition of the NER complex during individual steps are difficult to answer from classical bulk biochemical methods.

To address these questions, we previously initiated single molecule studies on the interaction of NER proteins with model DNA substrate by using scanning confocal fluorescence microscopy for investigations of XPA-DNA complexes embedded in agarose gel matrices [20]. Here FCS is presented as an alternative for the DNA-immobilization experiments performed in agarose. An important advantage of FCS lies in the absence of immobilization requirements. Consequently, both sample preparation and data acquisition are easier and faster, preserving the ability to measure individual molecular complexes.

It will be shown that FCS can rapidly screen and compare binding affinities of XPC-hHR23B protein complexes to different DNA substrates that model NER intermediates. The human XPC protein $(125 \mathrm{kD})$, which occurs in vivo always associated with the $58 \mathrm{kD}$ hHR23B protein, is known to be involved in the initial steps of damage recognition $[21,22]$. However, the repair process is strongly influenced by the mode of XPC-hHR23B binding, and the state of DNA required for specific binding of the protein complex is still not precisely understood [23]. Very recently, the crystal structure of the yeast XPC orthologue Rad4 bound to DNA was elucidated [24] and gave a better knowledge of the recognition mechanism. We have analyzed interactions of XPC-hHR23B protein complex with fluorescently labeled 90-bp double-stranded oligonucleotides (54 kD), (1) without any modification, (2) 
with a central 30-nt unpaired region and (3) containing a cholesterol modification. In addition, (4) the interaction with 90-mer single-stranded DNA (27 kD) was investigated. One DNA strand was 5'-labeled with a single Cy3.5 molecule to detect individual diffusing oligonucleotides. The mass difference between free DNA ( $27 \mathrm{kD}$ for ss DNA and $54 \mathrm{kD}$ for dsDNA) and XPC-hHR23B/DNA bound complex (210 or $237 \mathrm{kD}$ ) is sufficient to detect complex formation by FCS [12]. Comparison of the binding affinity of XPC-hHR23B to several DNA substrates does not require the measurement of a complete titration curve. Indeed, diffusion measurements suffice to determine the bound fractions in an excess of protein (protein-DNA 10:1 mixtures). An advantage of this strategy is the minimization of the number of required measurements. The diffusion time for free DNA and DNA/XPC-hHR23B was estimated from the application of Stokes-Einstein theory to oligonucleotides, despite the semi-flexible nature of the DNA oligomers [25], assuming a globular shape for oligonucleotides.

Our results demonstrate that FCS is a useful method for rapid determination of XPC-hHR23B binding affinity to different kind of DNA substrates modeling NER intermediates.

\section{Materials and methods}

\section{Material}

ssDNA 5'-Cy3.5 labeled 90 nucleotide (nt) single stranded DNA oligomers were purchased from DNA Technology A/ $\mathrm{S}$, Aarhus, Denmark. Cy3.5 was linked to the $5^{\prime}-\mathrm{OH}$ through a 3-carbon linker. The nucleotide sequence was according to reference [26].

dsDNA An unlabeled 90-nt strand of DNA complementary to the ssDNA was purchased from DNA Technology A/S, Aarhus, Denmark.

Bubble-DNA dsDNA with a central 30-nt unpaired (bubble) region was produced according to reference [26].

Cholesterol-damage DNA (Chol) A 90-nt ss-DNA, with a complementary sequence to the above mentioned ssDNA and a cholesterol modification [27] at position 60 from the 5 '-end (Eurogentec, Maastricht, the Netherlands) was purchased.

DNA annealing All ds-DNA substrates were prepared by mixing equimolar amounts of single strand oligomers in $25 \mathrm{mM}$ HEPES, $100 \mathrm{mM}$. NaCl, $10 \mathrm{mM}$. $\mathrm{MgCl}_{2}, \mathrm{pH} 7.5$ at a final concentration of $10^{-6} \mathrm{M}$. Hybridization was performed by heating the reaction mixture to $70{ }^{\circ} \mathrm{C}$ for 3 min followed by slow cooling to room temperature over several hours. Product formation was checked by electrophoresis [20] using an $8 \%$ non-denaturing polyacrylamide gel. No further purification was needed.

The XPC-hHR23B protein complex was obtained as described elsewhere [22] and used without any further purification.

\section{Sample preparation}

XPC-hHR23B/DNA mixtures were prepared at a molar ratio of 10:1. During incubation the XPC-hHR23B concentration was $10 \mathrm{nM}$ and the DNA concentration was $1 \mathrm{nM}$ in a $6 \mu \mathrm{l}$ volume of $25 \mathrm{mM}$ HEPES, $0.2 \mathrm{mM}$ EDTA, $120 \mathrm{mM} \mathrm{NaCl}, 20 \mathrm{mM} \mathrm{KCl}, 7 \mathrm{mM} \mathrm{MgCl}$, pH 7.5. Incubation was performed for $30 \mathrm{~min}$ at $30{ }^{\circ} \mathrm{C}$. For FCS measurements, the $6 \mu \mathrm{l}$ volume of solution (free DNA or mixture) was pipetted between two clean glass coverslips of $24 \mathrm{~mm}$ diameter and fitted in a sample holder. The coverslips were cleaned as follows: (1) sonication in $30 \%$ detergent (Hellmanex, Hellma) for $1 \mathrm{~h},(2)$ immersion in distilled water for $5 \mathrm{~min}$, (3) immersion in acetone for $15 \mathrm{~min}$, (4) immersion in distilled water for $5 \mathrm{~min}$, (5) immersion in $1 \mathrm{M}$ potassium hydroxide for $15 \mathrm{~min}$, (6) immersion in distilled water for $5 \mathrm{~min}$, (7) immersion in $1 \mathrm{M}$ potassium hydroxide for $15 \mathrm{~min}$ and (8) distilled water for $5 \mathrm{~min}$. The coverglasses were kept in nitric acid before use.

\section{Confocal microscopy setup}

We used a homemade confocal microscope for single molecule studies [20] in which excitation light is provided by a mixed $\mathrm{Ar}-\mathrm{Kr}$ gas laser (Innova 70; Coherent). The excitation light was focused onto the sample by a water immersion objective (C-Apochromat $63 \times, 1.20 \mathrm{NA}$; Zeiss). The reflected light was collected by the same objective and directed onto an avalanche photodiode (APD) device. A D605/55m emission filter was used for detection of Cy3.5 emission and a notch filter for suppression reflected $568 \mathrm{~nm}$ excitation wavelength (Chroma Technology Corp., Brattleboro, VT, USA). The selected emission is focused onto a $25 \mu \mathrm{m}$ pinhole located in front of the APD. The setup also offers imaging possibilities with a computer controlled piezoelectric scanning system. LabVIEW software (National Instruments, Austin, TX, USA) was used to program and perform signal acquisition and achieve time trace recording with a sampling time of $12.5 \mu \mathrm{s}$. 


\section{FCS measurements}

The FCS time-traces were recorded during $60 \mathrm{~s}$ with a $12.5 \mu$ s sampling rate, after which the autocorrelation functions were calculated. The laser intensity was $200 \mu \mathrm{W}$ at the sample. The autocorrelation was calculated directly from the recorded time-trace data with a homemade routine (Matlab 6.5: MathWorks, Inc., Natick, MA, USA). The autocorrelation trace was calculated at 7,000 lag times, with a minimum lag time of $12.5 \mu \mathrm{s}$, which corresponds to the sampling time. Quantitative analysis of the experimental autocorrelation function was performed using least-square analysis and Origin 6.0 software (Microcal Software, Inc., Nothampton, MA, USA). The calculation of the diffusion times required the $r$ and $z$ radii of the Gaussian confocal observation volume which were determined by imaging $6 \mathrm{~nm}$ gold beads to be respectively 0.24 and $1.05 \mu \mathrm{m}$ for our setup. The calibration of the FCS measurements was done with the dye rhodamine $6 \mathrm{G}$ which has a well-known diffusion coefficient [28].

The imaging capability of our setup allowed checking the homogeneity of the sample on a wide scale, especially for the absence of fluorescent aggregates able to diffuse or stick to cover-slips glass surfaces. Sample drying was assumed to be very limited, the total measurement time being around ten minutes. Comparison of successive autocorrelation curves served to check if any evolution with time occurred.

\section{Results}

\section{Autocorrelation curves}

Each cy3.5 labeled DNA substrate (ssDNA, dsDNA, Chol, bubble), was investigated free and mixed with the hHR23B-XPC protein complex at a 10:1 (protein/DNA) molar ratio to shift the equilibrium toward formation of DNA complexes. For any sample we recorded the fluctuations in photon count, due to fluorescence emission from individual molecules passing through the observation volume, as a function of time. In our conditions, with a confocal volume of $0.3 \mathrm{fl}$ and a concentration in fluorescent species of $1 \mathrm{nM}$, we have calculated an average number of molecules in the observation volume equal to 0.2 , being well in the single molecule regime. Autocorrelation analysis of these fluorescence fluctuations time traces consists of temporally correlating the recorded intensity signal according to Eq. 1.

$$
G(\tau)=\frac{\langle\delta F(t) \cdot \delta F(t+\tau)\rangle}{\langle F(t)\rangle^{2}}
$$

where $G(\tau)$ is the autocorrelation function, $\tau$ the lag time, $\delta F(t)$ is the fluctuation of fluorescence signal from the average $\langle F(t)>$ at a given time.

Figure 1 shows the normalized autocorrelation plots $G$ $(\tau)$ obtained for the four investigated DNA substrates, free and mixed with hHR23B-XPC. Each curve is the average of ten measurements; error bars are not represented for the sake of clarity, but are always smaller than $10 \%$ of the given autocorrelation coefficient. In all cases, the plots were significantly shifted toward larger lag times in the presence of hHR23B-XPC, indicating the presence of DNA-protein bound complexes. The characterization of this binding requires a quantitative analysis of FCS autocorrelation data. This was done with a homemade routine performing leastsquares fits of the experimental data according to the autocorrelation function describing translational diffusion through a Gaussian confocal volume element [9]:

$G(\tau)=N^{-1} \cdot\left(1+\tau / \tau_{d}\right)^{-1} \cdot\left(1+\tau /\left((z / r)^{2} \cdot \tau_{d}\right)\right)^{-1 / 2}$

where $\tau_{\mathrm{D}}$ is the diffusion time, i.e. the average time the molecules spend in the excitation volume, $z$ and $r$ are the radii of the Gaussian beam profile at $1 / e^{2}$ of its maximal intensity, and $N$ is the average number of fluorescent molecules simultaneously observed in the excitation volume.

Least-squares fitted curves of experimental data are represented by dotted-lines in Fig. 1, corresponding residual plots are shown as inserts. The apparently poor fit found for the few earliest data points did not affect the determination of diffusion times between 200 and $600 \mu \mathrm{s}$.

The obtained diffusion times of free DNA $\left(\tau_{\mathrm{d}}^{\text {free }}\right)$ and protein-DNA mixtures $\left(\tau_{\mathrm{d}}^{\text {mixt. }}\right)$ are given in Table 1 . Values are the mean $\pm \mathrm{SD}$ of ten measurements. Determined $\tau_{\mathrm{d}}^{\text {free }}$ for the double-stranded DNA substrates (dsDNA, Chol, bubble) are comparable, but it is interesting to note that perturbations of the semi-flexible structure of dsDNA lead to slightly faster diffusion time. A significantly faster diffusion time is found with free ssDNA, as expected due to its smaller molecular size and likely more globular shape. In all four cases studied, $\tau_{\mathrm{d}}^{\text {mixt }}$ values are significantly larger than corresponding $\tau_{\mathrm{d}}^{\text {free }}$. Thus, simple determination of $\tau_{\mathrm{d}}^{\text {free }}$ and $\tau_{\mathrm{d}}^{\text {mixt }}$ reveals DNA-protein interactions in the studied mixtures.

Estimate of the fraction of DNA bound

Equation 3 describes the extended autocorrelation function for two fluorescent diffusing species [9], here the free DNA with diffusion time $\tau_{\mathrm{d}}^{\text {free }}$ and the DNA/hHR23B-XPC 
Fig. 1 Autocorrelation plots (normalized to 1 at $12.5 \mu \mathrm{s}$ ) $G$ $(\tau)$ as a function of lag-time for the free dsDNA, ssDNA, Chol and bubble DNA substrates (open symbols) and their 1:10 mixtures with XPC-hHR23B protein complex (solid symbols). Dotted lines are the fits curves of experimental data obtained by application of the diffusion model described by Eq. 2. Residual plots corresponding to the difference between experimental and fits curves are shown as inserts in the lower part of each graph

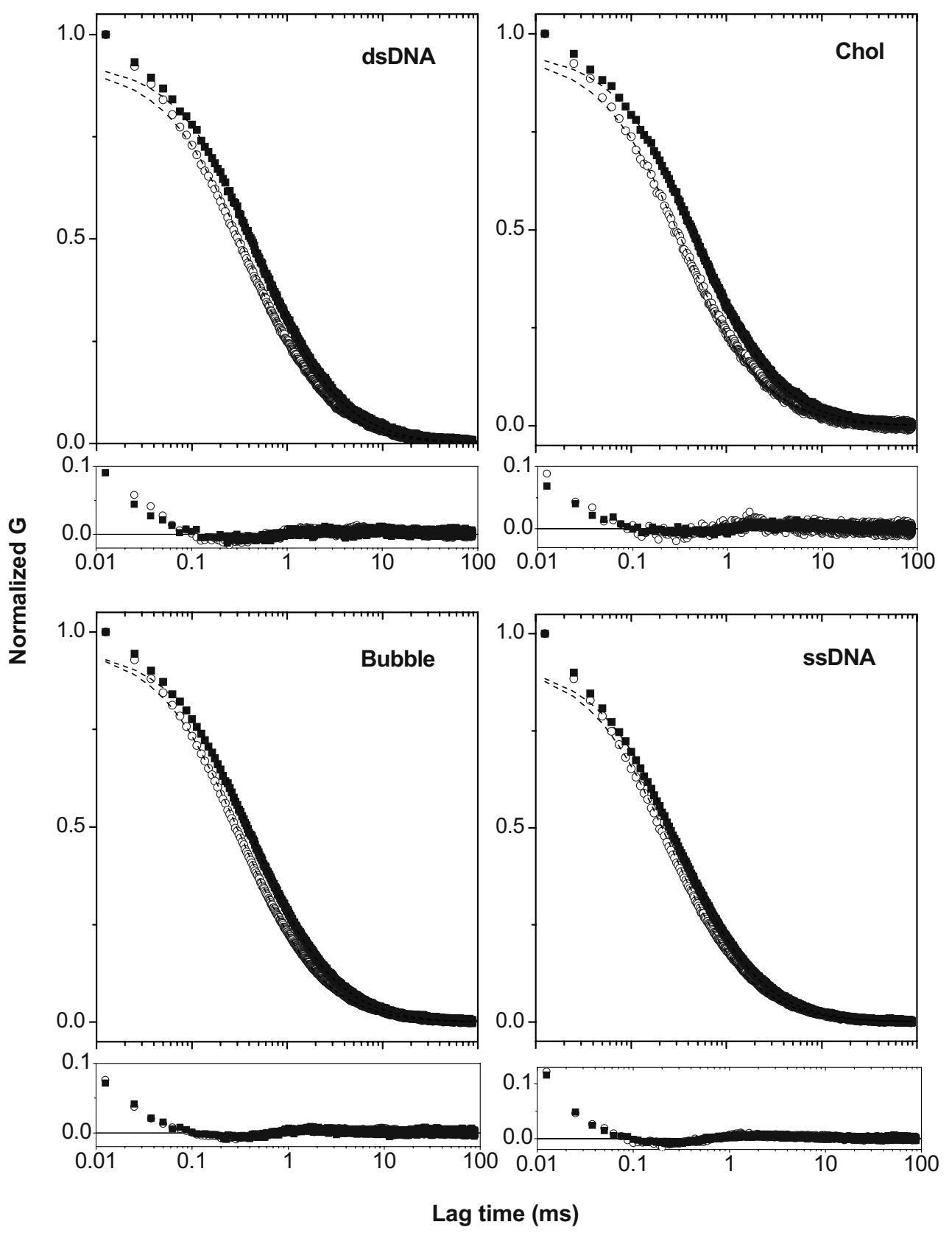

Table 1 Mean diffusion times $\left(\tau_{\mathrm{d}}\right)$ in $\mu$ s, degrees of binding $(\theta)$ and corresponding dissociation constants $\left(K_{\mathrm{D}}\right)$ in $\mathrm{nM}$, with $\mathrm{SD}$, obtained for the four investigated DNA substrates

\begin{tabular}{lcccc}
\hline & ssDNA & dsDNA & Chol & Bubble \\
\hline$\tau_{\mathrm{d}}^{\text {free }}$ & $258 \pm 13$ & $383 \pm 20$ & $350 \pm 24$ & $328 \pm 8$ \\
$\tau_{\mathrm{d}}^{\text {mixt }}$ & $320 \pm 12$ & $502 \pm 16$ & $500 \pm 26$ & $437 \pm 17$ \\
$\tau_{\mathrm{d}}^{\text {bind }}$ & $510 \pm 26$ & $629 \pm 34$ & $574 \pm 40$ & $538 \pm 14$ \\
$\Theta$ & $0.27 \pm 0.04$ & $0.58 \pm 0.09$ & $0.78 \pm 0.15$ & $3 \pm 1$ \\
$K_{\mathrm{D}}$ & $27 \pm 8$ & $7 \pm 3$ & $3 \pm 0.09$ \\
\hline
\end{tabular}

Diffusion times for free DNA $\left(\tau_{\mathrm{d}}^{\text {free }}\right)$ and DNA/XPC-hHR23B 1:10 mixtures $\left(\tau_{\mathrm{d}}^{\text {mixt }}\right)$ were experimentally determined, while $\tau_{\mathrm{d}}^{\text {bind }}$ was estimated from Eq. 4. The $\theta$ values were determined according to Eq. 3 and $K_{\mathrm{D}}$ values were estimated from the corresponding degrees of binding and protein concentration $[\mathrm{XPC}]=10 \mathrm{nM}: K_{\mathrm{D}}=(1-\theta) / \theta \times[\mathrm{XPC}]$. 
complex with diffusion time $\tau_{\mathrm{d}}^{\text {bind }}$. The fraction of DNA bound to protein is given by $\theta$. This treatment assumes that the quantum yield and the extinction coefficient of the fluorophore do not change upon binding.

$G(\tau)=\frac{1}{N} \cdot\left[\theta \cdot\left(1+\frac{\tau}{\tau_{\mathrm{d}}^{\text {bind }}}\right)^{-1} \cdot\left(1+\frac{\tau}{(z / r)^{2} \cdot \tau_{\mathrm{d}}^{\text {bind }}}\right)^{-1 / 2}+(1-\theta) \cdot\left(1+\frac{\tau}{\tau_{\mathrm{d}}^{\text {free }}}\right)^{-1} \cdot\left(1+\frac{\tau}{(z / r)^{2} \cdot \tau_{\mathrm{d}}^{\text {free }}}\right)^{-1 / 2}\right]$

The fraction of DNA bound $\theta$ can be obtained from this two-components diffusion model once $\tau_{\mathrm{d}}^{\text {free }}$ and $\tau_{\mathrm{d}}^{\text {bind }}$ have been determined.

The diffusion time for the different DNA substrates, $\tau_{\mathrm{d}}^{\text {free }}$, were directly obtained from the corresponding autocorrelation curves (Table 1). The diffusion time of the complex,, $\tau_{\mathrm{d}}^{\text {bind }}$, is unknown and its determination requires multiple measurements at different protein-DNA molar ratios. This method becomes inaccurate if multiple binding modes exist, i.e. if the studied protein is able to largely bind DNA in a non-specific manner as well, with multi-sites binding, or aggregation. Instead, we have chosen to estimate $\tau_{\mathrm{d}}^{\text {bind }}$ from the Stokes-Einstein relation, which holds strictly only for globular particles. In this way, $\tau_{\mathrm{d}}^{\text {bind }}$, $\tau_{\mathrm{d}}^{\text {free }}, m^{\text {free }}$ and $m^{\text {bind }}$ are related in the following way, and $\tau_{\mathrm{d}}^{\mathrm{bind}}$ can be determined:

$\frac{\tau_{\mathrm{d}}^{\text {bind }}}{\tau_{\mathrm{d}}^{\text {free }}}=\left(\frac{m^{\text {bind }}}{m^{\text {free }}}\right)^{\frac{1}{3}}$

where $m^{\text {free }}$ and $m^{\text {bind }}$ are masses for free DNA and DNAprotein complex respectively.

The estimations of $\tau_{\mathrm{d}}^{\text {bind }}$ are presented in Table 1 . The values for $\tau_{\mathrm{d}}^{\text {bind }}$ are systematically longer than $\tau_{\mathrm{d}}^{\text {mixt }}$, as must be expected from the fact that the percentage of DNAprotein complexes formed in the mixtures was lower than $100 \%$. The bound fraction $\theta$ was then obtained by performing least-squares fits of the autocorrelation traces according to Eq. 3, with the corresponding $\tau_{\mathrm{d}}^{\text {bind }}$ and $\tau_{\mathrm{d}}^{\text {free }}$ as fixed parameters. The resulting mean $\theta$ values \pm SD are depicted in Fig. 2 and in Table 1 with the estimated dissociation constants $\left(K_{\mathrm{D}}\right)$ for each investigated DNA substrate.

\section{Discussion}

Diffusion of free DNA substrates

In this study we have used FCS to investigate the interaction of the protein complex XPC-hHR23B which with three Cy3.5 labeled 90-bp double-stranded DNA substrates (dsDNA, Bubble, and Chol) and a 90-mer single-strand ssDNA. The determination of the bound fraction $\theta$ from FCS data relies on the validity of the Stokes-Einstein theory. The Stokes-Einstein theory implies a globular folded shape and a totally flexible 90-bp oligonucleotides. As expected, the ssDNA has the fastest diffusion not only for its smaller mass, but also for its likely globular shape [25]. Among the dsDNA substrates, bubble has the fastest mobility and this may also be caused by a relatively high flexibility. Finally, the Chol substrate seems to be slightly faster than the dsDNA, which again may point to a somewhat higher flexibility of this substrate compared to the undamaged one. Thus, dsDNAs clearly do not comply with the Stokes-Einstein criteria and will result in a significant change in their hydrodynamic radius and hence calculated $\tau_{\mathrm{d}}$. In other words, the theoretical $\tau_{\mathrm{d}}$ calculated from the mass would be smaller than the corresponding experimental value. This deviation from theory can be highlighted by comparing the cubic root of mass ratio $\left(\mathrm{m}^{\mathrm{ds}} / \mathrm{m}^{\mathrm{ss}}\right)^{1 / 3}$ which is 1.26 for dsDNA versus ssDNA, with the experimental $\tau_{\mathrm{d}}^{\mathrm{ds}} / \tau_{\mathrm{d}}^{\mathrm{ss}}$ ratios, when assuming the flexible nature of ssDNA [25]. Significant positive deviations were found for $\operatorname{dsDNA}\left(\tau_{\mathrm{d}}^{\mathrm{ds}} / \tau_{\mathrm{d}}^{\mathrm{ss}}=1.48\right)$ and Chol $\left(\tau_{\mathrm{d}}^{\mathrm{ds}} / \tau_{\mathrm{d}}^{\mathrm{ss}}=1.36\right)$, which must be understood as a deviation from this semi-flexible behavior for the respective dsDNA substrates. As already noted, the cholesterol

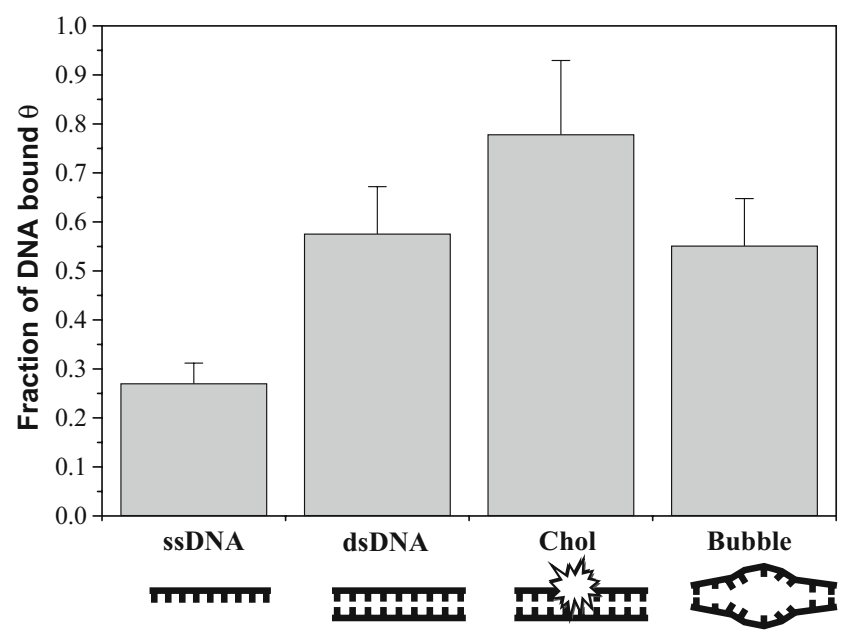

Fig. 2 Histogram plots of mean degrees of binding $\theta$, with error bars, for DNA/XPC-hHR23B 1:10 mixtures, calculated with $\mathbf{A}$ the two components diffusion model describes by Eq. 3. Schematic representations of DNA substrates are depicted 
modification seems to improve slightly the flexibility of the oligonucleotide. Finally, the ratio was similar to $\left(m^{\mathrm{ds}} / m^{\mathrm{ss}}\right)^{1 / 3}$ in the case of $30 \mathrm{bp}$ bubble DNA $\left(\tau_{\mathrm{d}}^{\mathrm{ds}} / \tau_{\mathrm{d}}^{\mathrm{ss}}=1.27\right)$, which would then indicate again that the large unpaired region improves flexibility and leads to a behavior of the $30 \mathrm{bp}$ bubble DNA more in agreement with Stokes-Einstein theory.

\section{Interaction of the ssDNA substrate with hHR23B-XPC}

The results show that the bound fraction for ssDNA $(0.27)$ is significantly weaker than that for double-stranded oligonucleotides, i.e. dsDNA (0.58), Chol (0.78) and $30 \mathrm{bp}$ bubble $(0.55)$. The binding affinity of XPC is thus clearly weaker for ssDNA than for the studied doublestranded oligonucleotides (dsDNA, Chol, bubble). In previous gel mobility shift assays [23, 29] a significantly higher XPC affinity for single-stranded DNA than for double-stranded DNA was observed. However, these same authors have noticed the poor affinity of XPC for singlestranded homopolymers and they have concluded that formation of secondary structures within heteropolymers single-stranded DNA could explain this tight binding. In our study we have avoided complementarity and we observe, therefore, a lower binding affinity XPC for ssDNA.

Binding specificity of the cholesterol modified DNA substrate

Among the DNA substrates, the Chol substrate exhibits the smallest XPC-hHR23B dissociation (binding coefficient equals to 0.78 ), while the bound fractions for dsDNA and bubble are similar (respectively 0.58 and 0.55 ). With electrophoretic gel-shift assays $K_{\mathrm{D}}$ values for XPChHR23B binding to undamaged 49-bp [30] and 60-bp [31] were determined to be $5 \mathrm{nM}$, in good agreement with our present results for dsDNA. Binding to UV-damaged DNA have resulted in dissociation constants of $0.12 \mathrm{nM}$ [30] for 49-bp DNA, $4 \mathrm{nM}$ [31] for 60-bp DNA, and $7 \mathrm{nM}$ [29] for 136-bp DNA. With fluorescence anisotropy measurements at equilibrium conditions $K_{\mathrm{D}}$ values of $10 \mathrm{nM}$ were obtained for binding to undamaged DNA and $1.1 \mathrm{nM}$ for binding to a 6-bp unpaired region within 36-bp double-strand DNA [32]. We conclude that the binding coefficient determined by FCS is in agreement with results obtained with other methods.

The role of XPC in primary recognition of damaged DNA sites, suggests that strong damage discrimination could be expected. However, our data shows an avidity of XPC for undamaged DNA that is only about 0.7 times smaller than for damaged substrates. Similar observations are extensively reported in literature (see [33] for a review) and it was suggested that non-specific binding to undamaged dsDNA substrates may occur due to a preferential affinity of the XPC-hHR23B complex to the DNA ends [32]. A recent work with the Rad4 homologue [24] suggests that nearly all contacts are made all along the DNA backbone, even for undamaged substrates. Another non-specific contribution may be short lifetime low affinity complexes that can be detected by FCS. Indeed, a model has proposed simple stochastic diffusion of proteins to be a key in DNA-protein contacts and subsequent regulation of nuclear processes [34]. Finally, we note that multiple XPC binding to DNA substrates, that would biased the results, could be ruled out here due to steric limitations.

Role of DNA unpaired region in interaction with DNA-XPC-hHR23B

It is interesting to note that the comparison of degrees of binding for dsDNA and bubble substrates indicates that both would experience the same affinity with XPChHR23B protein complex. At first sight, this seems to be in conflict with some findings that claimed for structurespecific damage recognition, where XPC would preferentially recognize and bind to double-stranded/single-stranded junctions [23] as found in the bubble substrate. On the basis of NMR experiments, other researchers have recently suggested that XPC damage recognition results from searching for unusual groove width, distortion and/or bent of helix [35]. However, a scanning force microscopy study [36] has suggested that the XPC specific binding to DNA induces a distortion in such a way that the interaction at the damaged site would therefore lead to a more stable complex than on undamaged DNA. Our data supports the notion that, despite the presence of both helix distortion and single-stranded region within a 90-bp DNA with a 30-nt central unpaired region (bubble), this substrate is unlikely to specifically bind XPC. We can propose that the size of the bubble is such that the DNA structure prevents stable specific binding to XPC. Indeed, previously cited studies have mainly utilized DNA substrates with small mismatches $(<10-b p)$. Our findings are thus consistent with the notion that XPC is not part of the open NER complex in which the DNA is thought to be opened for about 30-nt by the TFIIH helicase action prior to incision. As a perspective, study of small mismatches bubble substrates, containing or not a cholesterol modification, should provide additional information about the capability of a damage to stabilize specific XPC-DNA binding.

To conclude, we have shown that FCS can be successfully used to determine protein binding affinity and specificity for DNA substrates. We have obtained significant variations in DNA diffusion time in the presence of an excess of protein, and binding parameters have been 
estimated from FCS data to show a strong affinity between the XPC protein and DNA substrate containing Chol damage. Therefore, our results show that FCS is a convenient method, requiring low amounts of material, for fast comparison of various DNA substrates in DNA-protein binding experiments.

Acknowledgement This work was supported by the Dutch Technology Foundation (STW) through grant TTN.4821 and the European Community through a Human Frontiers in Science Program grant RGP0007/2004-C.

Open Access This article is distributed under the terms of the Creative Commons Attribution Noncommercial License which permits any noncommercial use, distribution, and reproduction in any medium, provided the original author(s) and source are credited.

\section{References}

1. Michalet X, Weiss S (2002) Single-molecule spectroscopy and microscopy. Comptes Rendus Physique 3(5):619-644

2. Bohmer M, Enderlein J (2003) Fluorescence spectroscopy of single molecules under ambient conditions: methodology and technology. Chemphyschem 4(8):793-808

3. Moerner WE, Fromm DP (2003) Methods of single-molecule fluorescence spectroscopy and microscopy. Rev Sci Instrum 74 (8):3597-3619

4. Van Craenenbroeck E, Engelborghs Y (2000) Fluorescence correlation spectroscopy: molecular recognition at the single molecule level. J Mol Recognit 13(2):93-100

5. Haustein E, Schwille P (2004) Single-molecule spectroscopic methods. Curr Opin Struct Biol 14(5):531

6. Krichevsky O, Bonnet G (2002) Fluorescence correlation spectroscopy: the technique and its applications. Rep Prog Phys 65 (2):251-297

7. Elson EL, Madge D (1974) Fluorescence correlation spectroscopy: I. Conceptual basis and theory. Biopolymers 13:1-27

8. Rigler R, Mets U, Widengren J, Kask P (1993) Fluorescence correlation spectroscopy with high count rate and low-background-analysis of translational diffusion. Eur Biophys J 22 (3):169-175

9. Haustein E, Schwille P (2003) Ultrasensitive investigations of biological systems by fluorescence correlation spectroscopy. Methods 29(2):153-166

10. Hess ST, Webb WW (2002) Focal volume optics and experimental artifacts in confocal fluorescence correlation spectroscopy. Biophys J 83(4):2300-2317

11. Webb WW (2001) Fluorescence correlation spectroscopy: inception, biophysical experimentations, and prospectus. Appl Opt 40 (24):3969-3983

12. Meseth U, Wohland T, Rigler R, Vogel H (1999) Resolution of fluorescence correlation measurements. Biophys J 76(3):1619-1631

13. Sevenich FW, Langowski J, Weiss V, Rippe K (1998) DNA binding and oligomerization of $\mathrm{NtrC}$ studied by fluorescence anisotropy and fluorescence correlation spectroscopy. Nucleic Acids Res 26(6):1373-1381

14. Xu H, Frank J, Trier U, Hammer S, Schroder W, Behlke J, Schafer-Korting M, Holzwarth JF, Saenger WU (2001) Interaction of fluorescence labeled single-stranded DNA with hexameric DNA-helicase RepA: a photon and fluorescence correlation spectroscopy study. Biochemistry 40(24):7211-7218

15. Schubert F, Zettl H, Hafner W, Krauss G, Krausch G (2003) Comparative thermodynamic analysis of DNA-protein interactions using surface plasmon resonance and fluorescence correlation spectroscopy. Biochemistry 42(34):10288-10294

16. Wolcke J, Reimann M, Klumpp M, Gohler T, Kim E, Deppert WU (2003) Analysis of p53 "latency" and "activation" by fluorescence correlation spectroscopy. Evidence for different modes of high affinity DNA binding. J Biol Chem 278 (35):32587-32595

17. LeCaptain DJ, Michel MA, Van Orden A (2001) Characterization of DNA-protein complexes by capillary electrophoresis-single molecule fluorescence correlation spectroscopy. Analyst 126 (8): $1279-1284$

18. Kobayashi T, Okamoto N, Sawasaki T, Endo Y (2004) Detection of protein-DNA interactions in crude cellular extracts by fluorescence correlation spectroscopy. Anal Biochem 332(1):58-66

19. Hoeijmakers JH (2001) Genome maintenance mechanisms for preventing cancer. Nature 411(6835):366

20. Segers-Nolten GMJ, Wyman C, Wijgers N, Vermeulen W, Lenferink ATM, Hoeijmakers JHJ, Greve J, Otto C (2002) Scanning confocal fluorescence microscopy for single molecule analysis of nucleotide excision repair complexes. Nucleic Acids Res 30(21):4720-4727

21. Sugasawa K, Okamoto T, Shimizu Y, Masutani C, Iwai S, Hanaoka F (2001) A multistep damage recognition mechanism for global genomic nucleotide excision repair. Genes \& Development 15(5):507

22. Sugasawa K, Ng JMY, Masutani C, Iwai S, van der Spek PJ, Eker APM, Hanaoka F, Bootsma D, Hoeijmakers JHJ (1998) Xeroderma pigmentosum group $\mathrm{C}$ protein complex is the initiator of global genome nucleotide excision repair. Mol Cell 2(2):223232

23. Sugasawa K, Shimizu Y, Iwai S, Hanaoka F (2002) A molecular mechanism for DNA damage recognition by the xeroderma pigmentosum group C protein complex. DNA Repair 1(1):95107

24. Min J, NP P (2007) Recognition of DNA damage by the Rad4 nucleotide excision repair protein. Nature 449(7162):570-U7

25. Shusterman R, Alon S, Gavrinyov T, Krichevsky O (2004) Monomer dynamics in double-and single-stranded DNA polymers. Phys Rev Lett 92(4):048303

26. Matsunaga T, Park CH, Bessho T, Mu D, Sancar A (1996) Replication protein A confers structure-specific endonuclease activities to the XPF-ERCC1 and XPG subunits of human DNA repair excision nuclease. J Biol Chem 271:11047-11050

27. Mu D, Hsu DS, Sancar A (1996) Reaction mechanism of human DNA repair excision nuclease. J Biol Chem 271:8285-8294

28. Jaffiol R, Blancquaert Y, Delon A, Derouard J (2006) Spatial fluorescence cross-correlation spectroscopy. Appl Opt 45:12251235

29. Batty D, Rapic'-Otrin V, Levine AS, Wood RD (2000) Stable binding of human XPC complex to irradiated DNA confers strong discrimination for damaged sites. J Mol Biol 300(2):275290

30. Wakasugi M, Sancar A (1999) Order of assembly of human DNA repair excision nuclease. J Biol Chem 274(26):18759-18768

31. Reardon JT, Mu D, Sancar A (1996) Overproduction, purification, and characterization of the XPC subunit of the human DNA repair excision nuclease. J Biol Chem 271(32):19451

32. Hey T, Lipps G, Sugasawa K, Iwai S, Hanaoka F, Krauss G (2002) The XPC-HR23B complex displays high affinity and 
specificity for damaged DNA in a true-equilibrium fluorescence assay. Biochemistry 41(21):6583-6587

33. Thoma BS, Vasquez KM (2003) Critical DNA damage recognition functions of XPC-hHR23B and XPA-RPA in nucleotide excision repair. Mol Carcinog 38(1):1-13

34. Houtsmuller AB, Vermeulen W (2001) Macromolecular dynamics in living cell nuclei revealed by fluorescence redistribution after photobleaching. Histochem Cell Biol 115(1):13-21
35. Lee JH, Park CJ, Shin JS, Ikegami T, Akutsu H, Choi BS (2004) NMR structure of the DNA decamer duplex containing double T center dot $\mathrm{G}$ mismatches of cis-syn cyclobutane pyrimidine dimer: implications for DNA damage recognition by the XPC-hHR23B complex. Nucleic Acids Res 32(8):2474-2481

36. Janicijevic A, Sugasawa K, Shimizu Y, Hanaoka F, Wijgers N, Djurica M, Hoeijmakers JHJ, Wyman C (2003) DNA bending by the human damage recognition complex XPC-HR23B. DNA Repair 2(3):325-336 